\title{
Avaliação da usabilidade do geoportal da infraestrutura de dados espaciais da Bahia (IDE-BA)
}

\author{
Elaine Gomes Vieira de Jesus ${ }^{1}$ \\ Patricia Lustosa Brito ${ }^{2}$ \\ Vivian de Oliveira Fernandes ${ }^{2}$
}

Spatial data insfrastructures (SDI-BA) geoportal usability evaluation

Recebido em abril de 2018.

Aprovado em novembro de 2018.

\begin{abstract}
RESUMO
As Infraestruturas de Dados Espaciais (IDE) representam ferramentas a partir das quais as instituições participantes disponibilizam os dados geoespaciais que produzem por meio de geoserviços. As IDEs possuem Geoportais e visualizadores de mapas que permitem o acesso do usuário aos dados. Sendo assim, este artigo avalia critérios de Usabilidade do Geoportal e Visualizador de Mapas da Infraestrutura de Dados Espaciais do Estado da Bahia (IDE Bahia). O teste de usabilidade foi realizado em setembro de 2017 com seis usuários de informações geoespaciais, professores de ensino superior e alunos de pós-graduação, da Universidade Federal da Bahia (UFBA) com o objetivo de avaliar critérios de usabilidade e mensurar a satisfação do usuário. Verificou-se que dentre as 7 tarefas básicas analisadas apenas duas atenderam plenamente os usuários, as demais 50\% ou mais dos usuários não completaram as tarefas e se posicionaram insatisfeitos com a ferramenta. Com base nisso, o artigo apresenta alguns pontos a serem melhorados, para permitir uma interação mais eficiente e eficaz deste sistema com o usuário.
\end{abstract}

PALAVRAS-CHAVE: Infraestrutura de Dados Espaciais (IDE). Visualizador de mapas. Testes de Usabilidade.

\begin{abstract}
Spatial Data Infrastructures (SDI) represent tools from which participating institutions provide the geospatial data they produce through geoservices. The SDIs have Geoportals and map viewers that allow users to access the data. This article evaluates the usability criteria of the Geoportal and Map
\end{abstract}

\footnotetext{
${ }^{1}$ Programa de Pós-Graduação em Arquitetura e Urbanismo, Universidade Federal da Bahia, Brasil. Email: elainegomes623@gmail.com

${ }^{2}$ Programa de Pós-Graduação em Engenharia Civil, Universidade Federal da Bahia, Brasil. E-mail: britopatricia@hotmail.com, vivian.fernandes@ufba.br
} 
Viewer from the Spatial Data Infrastructure of the State of Bahia (IDE Bahia). The usability test was conducted in September 2017 with six users of geospatial information, teachers and graduate students, from the Federal University of Bahia (UFBA). The objective was to evaluate the usability criteria and to measure user's satisfaction. It was verified that all users completed successfully only two tasks, out of the 7 basic tasks demanded, $50 \%$ or more of the users did not complete the other tasks and declared to be unsatisfied with the tool. Based on this, the article presents users main issues, pointing out improvements opportunities to allow a more efficient and effective interaction of this system with the user.

KEYWORDS: Spatial Data Infrastructure (SDI). Map Viewer. Usability Tests.

$$
* * *
$$

\section{Introdução}

Com o rápido avanço das tecnologias de produção de dados e de disseminação destes na internet torna-se necessário investir em formas de acesso e disponibilização ao usuário. Com este intuito surgem as iniciativas de Infraestruturas de Dados Espaciais (IDE) que possibilitam ao usuário ter acesso aos dados e metadados de diversas instituições que as compõem de forma mais eficiente e eficaz. Entretanto é necessário seguir normas, padrões e políticas (ou acordos de gestão) para produção, atualização, compartilhamento e disponibilização dos dados.

Dados e metadados (informações sobre quem produziu o dado, com que finalidade, escala, abrangência espacial, período de levantamento e elaboração, etc.) são componentes básicos de uma Infraestrutura de Dados Espaciais. Em uma IDE os usuários tem acesso aos metadados por meio de um Geoportal que representa um portal web que possibilita visualização, edição e análise dos dados através de geoserviços. Os geoportais também possuem visualizadores de mapas para representar os resultados das buscas e interações realizadas pelo usuário.

Apesar dos geoserviços representarem uma maneira de simplificar o acesso aos dados geoespaciais de forma eficiente e eficaz nem sempre as Infraestruturas de Dados Espaciais conseguem atender as necessidades dos

Rev. Bras. de Cartografia, vol. 70, Edição Especial "XXVII Congresso Brasileiro de 
usuários. Sendo assim, o objetivo deste artigo é realizar testes para avaliar a usabilidade do Geoportal e do visualizador de mapas da IDE-BA através das ferramentas e dos geoserviços existentes.

Em relação aos geoserviços de uma IDE, foram considerados neste artigo o Web Map Service (WMS) que permite apenas a visualização ao mapa em forma de figura e o Web Feature Service (WFS) que oferece acesso à informação ao nível de feições e suas propriedades pois são os geoserviços encontrados na IDE-Bahia.

\section{Infraestrutura de Dados Espaciais (IDE)}

Uma Infraestrutura de Dados Espaciais representa um conjunto de tecnologias, políticas e mecanismos institucionais que facilita o acesso aos dados espaciais. A IDE fornece uma base para a busca de dados espaciais e aplicações para usuários e fornecedores de todos os níveis do governo, setores comerciais, setores sem fins lucrativos, das universidades e dos cidadãos em geral. Deve incluir também os critérios organizacionais necessários para coordenar e administrar essa infraestrutura nas escalas locais, nacionais ou transnacionais (GSDI, 2004, p. 8).

Paixão et al. (2008), ressaltam que um dos principais objetivos da IDE é minimizar a duplicação de ações em busca de dados. Este objetivo é alcançado através da padronização e compartilhamento de dados. Assim sendo, o papel da IDE é facilitar o acesso aos dados, permitindo ao usuário saber os dados que são produzidos e/ou disponibilizados por determinada instituição, bem como, quais são as condições de acesso e uso dessas informações, através de um portal único.

Os metadados são componentes importantes de uma IDE. Eles agrupam informações como escala do dado, ano de elaboração e atualização, empresa responsável por sua criação, sistema geodésico de referência, dentre outros. 
Segundo Lisboa Filho et al. (2013), os metadados desempenham uma função primordial dentro de uma IDE, pois garantem o cumprimento do seu principal objetivo, que é tornar os dados e serviços geoespaciais acessíveis aos usuários.

Nogueras - ISO et al. (2005), destacam que a obtenção do dado através de uma IDE, disponibilizada na Web, é realizada por meio da busca em catálogos de metadados, que por sua vez, facilitam a descoberta e recuperação. Nesses ambientes os dados são obtidos por meio de download ou através de geoserviços.

Em relação aos geoserviços de uma IDE se destacam o Web Map Service (WMS) e o Web Feature Service (WFS). O WMS baseado no padrão ISO 19128, permite apenas a visualização ao mapa solicitado em forma de figura, em formato PNG, GIF ou JPEG, ou seja, geralmente não possibilita o uso dos dados vetoriais e matriciais, enquanto o WFS (ISO 19142) oferece ao usuário acesso à informação ao nível de feições e suas propriedades (CAMBOIM, 2013). Os metadados seguem padronizações de órgãos internacionais como a International Organization for Standardization (ISO). Dentre os padrões de metadados, destaca-se o padrão ISO19115 (ISO, 2003).

Lisboa Filho et al. (2013), indicam que o padrão ISO19115 abrange vários outros padrões de metadados, tornando-se um superconjunto de alguns deles. Devido ao fato de ser um padrão extenso e muito complexo (mais de 300 elementos de metadados) o próprio padrão indica que na maioria das situações nem todos os elementos são necessários. Sendo assim, um grupo de 20 metadados foi definido como sendo o núcleo do padrão, o que significa que estes elementos devem ser mantidos em todos os perfis definidos com base no padrão ISO19115.

2.1 Infraestrutura de dados espaciais do estado da Bahia

O Decreto N 10.185 (BAHIA, 2006) instituiu o Portal de Informações Geoespaciais do Estado da Bahia GEOPORTAL Bahia, destinado a: 
a) coletar, tratar, compartilhar e disponibilizar bases espaciais de órgãos e entidades do Poder Executivo Estadual;

b) promover o intercâmbio de dados e o acesso a informações espaciais produzidos por outros Poderes e Esferas de Governo, por organismos não governamentais e pela iniciativa privada;

Este decreto foi considerado durante o processo de estabelecimento, o marco legal da Infraestrutura de Dados Espaciais da Bahia (IDE-Bahia) servindo de suporte legal e institucional para as ações de organização, consolidação e publicação de dados geoespaciais (BAHIA, 2016).

Entretanto a Infraestrutura de Dados Espaciais do Estado da Bahia IDE-BA foi instituída apenas nove anos depois, através do decreto ${ }^{\circ} 12.219$ de 24 de julho de 2015 (BAHIA, 2015). Esta IDE abrange dados e metadados das instituições consideradas "nós pioneiros". São elas: Superintendência de Estudos Econômicos e Sociais da Bahia (SEI), Companhia de Desenvolvimento Urbano do Estado da Bahia (CONDER), Instituto do Meio Ambiente e Recursos Hídricos (Inema), Empresa Baiana de Águas e Saneamento S. A (Embasa), Secretaria de Segurança Pública (SSP), Empresa Baiana de Desenvolvimento Agrícola (EBDA) e Departamento de Infraestrutura de Transportes da Bahia (DERBA). No entanto, essas duas últimas instituições foram extintas pela lei n 13.204 (BAHIA, 2014).

O decreto $n^{\circ} 12.219$ (BAHIA, 2015), determinou que a gestão da IDE Bahia ficaria a cargo da Superintendência de Estudos Econômicos e Sociais da Bahia (SEI), como Secretaria Executiva da Comissão Estadual de Cartografia (CECAR), órgão colegiado da Secretaria do Planejamento (SEPLAN). Sendo assim, a SEI tornou-se responsável por implantar, manter e gerir a estrutura tecnológica do nó central da IDE-Bahia; bem como implantar, manter e gerir o Geoportal Bahia, garantindo os recursos dedicados e incorporando novas funcionalidades (BAHIA, 2015).

O funcionamento da IDE-Bahia considera a estrutura apresentada na Figura 1, na qual o Geoportal acessa o catálogo de serviços web e pesquisa em uma base de dados central os metadados dos dados disponibilizados por cada 
"instituição nó". Esta base central captura, através de serviço web, os metadados cadastrados em cada nó, sincronizando as informações e mantendo-as disponíveis ao público de maneira única (SOUZA, et al., 2015).

Figura 1 - Arquitetura da IDE - Bahia.

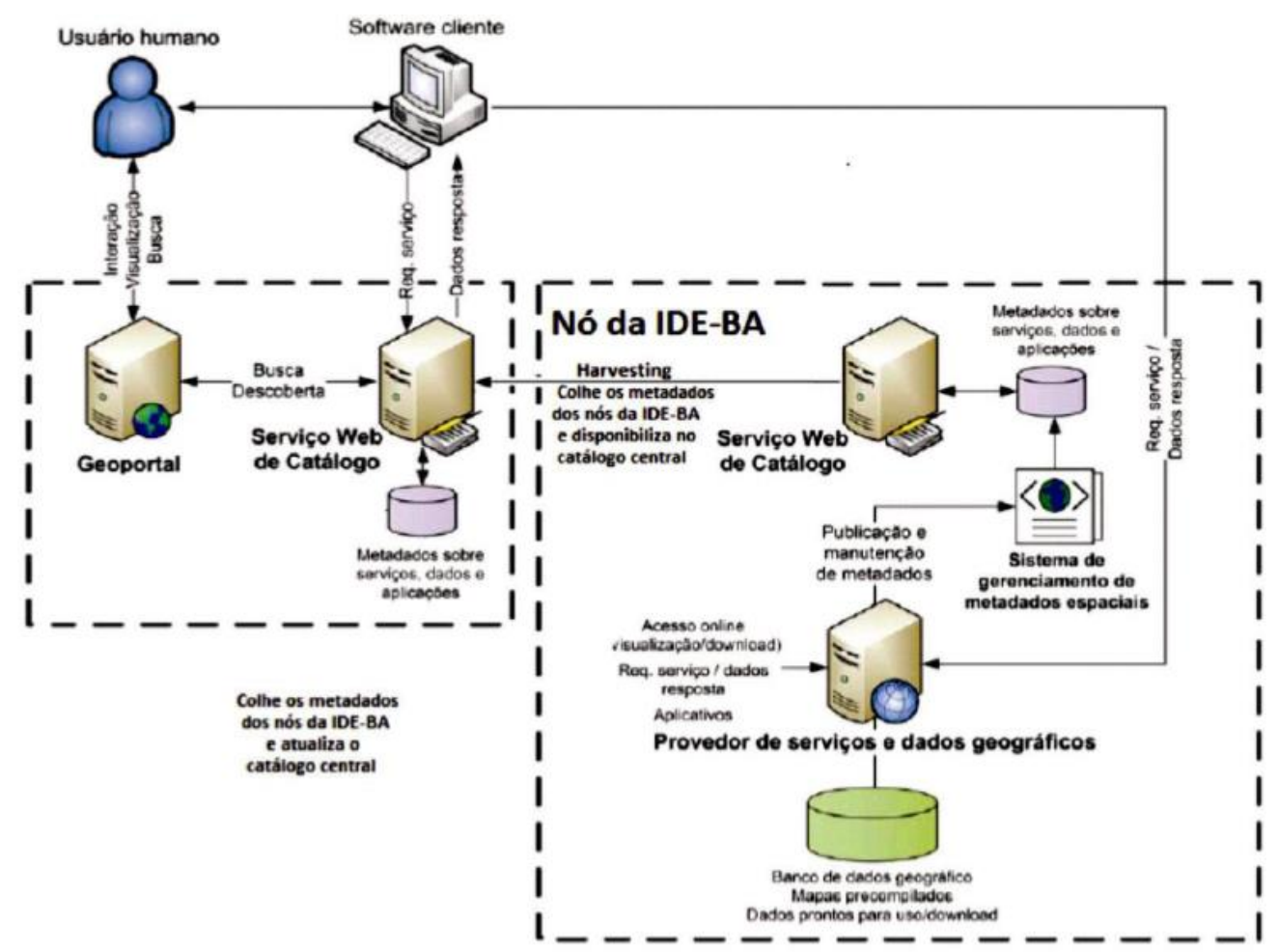

Fonte: Bahia (2017) apud Davis (2010) e S\&C (2014).

\section{Usabilidade}

Usabilidade é um termo que pode ser aplicado ao uso de interfaces web. É possível medi-la através do cumprimento de tarefas de modo eficiente e eficaz pelo usuário, por meio da mínima quantidade de erros e pela satisfação subjetiva do mesmo. Como alerta Wincler e Pimenta (2002), torna-se necessário considerar questões de usabilidade para o desenvolvimento de interfaces web, a fim de minimizar problemas encontrados durante a interação com usuário.

Uma interface pode ser avaliada com métodos e técnicas com ou sem a presença do usuário. Dentre os métodos e técnicas sem a presença do usuário,

Rev. Bras. de Cartografia, vol. 70, Edição Especial "XXVII Congresso Brasileiro de 
mais utilizados em trabalhos científicos, destacam-se: as inspeções por guidelines e checklist, avaliação heurística e critérios ergonômicos.

O método de avaliação heurística é um dos métodos mais utilizados para avaliação de interfaces em decorrência da facilidade de sua aplicação; envolve dez princípios que foram propostos por Nielsen (1993), conforme Quadro 1. Neste método cada avaliador realiza uma inspeção gerando como resultado uma lista de problemas de usabilidade, que indica os princípios que foram violados e a gravidade do problema.

Quanto aos métodos de avaliação com o usuário, destaca-se o teste de usabilidade, que tem o objetivo de identificar pontos críticos na interação do mesmo com um sistema, que podem consistir em problemas de usabilidade. Este método é aplicado com um número pequeno de usuários, geralmente com 5 usuários já é possível identificar aproximadamente $75 \%$ dos problemas mais críticos de uma interface (NIELSEN, 1993).

Dias (2003), salienta que os testes de usabilidade podem ser utilizados em qualquer fase de elaboração e implantação da interface. Quando usado na fase inicial de desenvolvimento do sistema, consegue identificar os parâmetros ou elementos que deverão ser implantados. Já na fase intermediária, funciona como validação ou refinamento do projeto e, na fase final, busca confirmar se o sistema atende aos objetivos e necessidades dos usuários.

Muitas técnicas podem ser utilizadas em conjunto na realização dos testes como: o estímulo ao usuário para verbalizar suas ações conhecidas como Think Aloud (pensar em voz alta), a mensuração por desempenho, aplicação de questionários e entrevistas e o uso de sistemas espiões que gravam os comandos do usuário durante as interações. Assim, problemas de usabilidade são identificados quando se observa usuários com dificuldades para concluir suas tarefas. 
Quadro 1 - Heurísticas propostas por Nielsen (1993).

\begin{tabular}{|c|c|}
\hline PRINCÍPIOS & DESCRIÇÃO \\
\hline 1- Visibilidade do estado do sistema & $\begin{array}{c}\text { O sistema deve sempre manter o usuário informado } \\
\text { sobre o que está acontecendo, através de feedback } \\
\text { em tempo razoável. }\end{array}$ \\
\hline $\begin{array}{l}\text { 2- Compatibilidade entre o sistema e o } \\
\text { mundo real }\end{array}$ & $\begin{array}{l}\text { O sistema deve falar a linguagem dos usuánios, } \\
\text { com palavras, frases e conceitos que sejam } \\
\text { familiares para ele, em vez de termos técnicos. } \\
\text { Deve seguir as convenções do mundo real, fazendo } \\
\text { com que informações apareçam de forma natural } \\
\text { seguindo uma ordem lógica. }\end{array}$ \\
\hline 3- Liberdade e controle do usuánio & $\begin{array}{c}\text { Se o usuário cometer algum engano na escolha das } \\
\text { opções, o sistema deverá indicar a maneira de sair } \\
\text { da situação indesejada, sendo possível refazer e } \\
\text { desfazer operações com facilidade. }\end{array}$ \\
\hline 4- Consistência e padrões & $\begin{array}{l}\text { O sistema deve seguir convenções, a fim de evitar } \\
\text { que o usuánio tenha que adivinhar o significado das } \\
\text { ações e diálogos. }\end{array}$ \\
\hline 5- Prevenção de erros & $\begin{array}{l}\text { O sistema deve prever possiveis erros cometidos } \\
\text { pelos usuánios. Se isso não for possivel, deve } \\
\text { fornecer mensagens que possibilitam o tratamento } \\
\text { do erro. }\end{array}$ \\
\hline $\begin{array}{c}\text { 6-Reconhecimento ao invés de } \\
\text { Recordação }\end{array}$ & $\begin{array}{l}\text { Minimizar a carga de memória do usuánio, fazendo } \\
\text { com que objetos, ações e opções estejam visíveis. } \\
\text { O usuánio não deve ter que lembrar de informações } \\
\text { de uma parte do diálogo para outro. }\end{array}$ \\
\hline $\begin{array}{c}\text { 7- Flexibilidade e eficiência na } \\
\text { utilização }\end{array}$ & $\begin{array}{l}\text { Aceleradores de interação, invisiveis para usuánios } \\
\text { iniciantes e possibilidade de criar atalhos para } \\
\text { ações frequentes são recursos apropriados para } \\
\text { todos os usuários, independente da sua experiência } \\
\text { com o sistema. }\end{array}$ \\
\hline 8- Estética e design & $\begin{array}{c}\text { Os diálogos só devem conter informações } \\
\text { relevantes. }\end{array}$ \\
\hline $\begin{array}{l}\text { 9- Suporte aos usuários (em relação } \\
\text { aos erros) }\end{array}$ & $\begin{array}{l}\text { As mensagens de enro devem ser expressas em } \\
\text { linguagem simples, indicar claramente o problema } \\
\text { e sugerir uma solução. }\end{array}$ \\
\hline 10- Ajuda e documentação & $\begin{array}{l}\text { A opção de ajuda do sistema deve estar sempre } \\
\text { disponível, mesmo quando o sistema é fácil de } \\
\text { usar. Esta opção deve ser fácil de localizar e deve } \\
\text { ser focada nas tarefas do usuánio. }\end{array}$ \\
\hline
\end{tabular}

Fonte: Adaptado de Nielsen (1993).

De acordo com Wincler e Pimenta (2002), a observação do avaliador durante a realização dos testes pode ocorrer de maneira direta, observando os usuários na execução das tarefas frente a frente ou por meio dos espelhos falsos que não permitem ao usuário ver o avaliador. As informações podem ser registradas por meio de anotações do avaliador ou através de câmeras ou gravadores de áudio. Estes registros permitirão ao avaliador identificar e discutir os problemas de usabilidade encontrados na interação. Sugere-se a utilização de duas câmeras, uma para gravar as reações do usuário e a outra para registrar os comandos realizados pelo usuário por meio da tela do computador.

Rev. Bras. de Cartografia, vol. 70, Edição Especial "XXVII Congresso Brasileiro de 
Alguns questionários também podem ser utilizados para avaliar uma interface web e medir a satisfação do usuário ao desenvolver tarefas num sistema, como o Software Usability Measurement Inventory (SUMI), Web Local Analysis and Inventory of Measure (WAMMI), Questionnaire for User Interaction Satisfaction (QUIS) e o NASA-TLX. Este último é um questionário que abrange parâmetros subjetivos e tem como objetivo mensurar a carga de trabalho (demanda mental, demanda física, demanda temporal, performance, esforço e nível de frustração) de um usuário ao desempenhar determinada tarefa.

Desta forma, os testes de usabilidade podem identificar critérios qualitativos através da mensuração por desempenho e satisfação subjetiva dos usuários e também critérios qualitativos. Dentre estes, Nielsen (1993) destaca:

a) o tempo que os usuários levam para concluir uma tarefa;

b) o número de tarefas de vários tipos que podem ser concluídas, dado um limite de tempo;

c) a taxa entre interações realizadas com sucesso e os erros;

d) o número de erros do usuário;

e) o número de comandos ou outras funcionalidades que foram usadas;

f) o número de comandos ou outras funcionalidades que nunca foram usadas;

g) o número de ferramentas que o usuário é capaz de lembrar, após executar as tarefas;

h) o número de vezes que o usuário expressa insatisfação ou contentamento;

i) a proporção de usuários que usam estratégias eficientes e eficazes de trabalho em comparação com aqueles que usam estratégias ineficientes e ineficazes. 


\section{Acesso aos Dados Geoespaciais e sua Disponibilização}

Os dados e informações geoespaciais são elementos que subsidiam o processo de tomada de decisão por gestores em diferentes esferas do poder. No entanto existem alguns desafios principalmente no acesso e disponibilização deste dado no Brasil. Desta forma algumas pesquisas têm sido realizadas para compreender essa dinâmica.

Jesus (2015), realizou um estudo para identificar os problemas encontrados no acesso e disponibilização de dados através de entrevistas realizadas com docentes de ensino superior da Universidade Federal da Bahia. As entrevistas mostraram que as principais dificuldades foram:

a) em relação à aquisição dos dados oficiais produzidos por órgãos públicos ou mesmo na universidade (produzidos por projetos ou pesquisas de mestrado e doutorado) e sua confiabilidade (metadados). Verificou-se que os usuários não costumam enviar um ofício para as instituições solicitando os dados pelo fato de levar um tempo maior para adquiri-los então acabam obtendo-os através de terceiros (dados que muitas vezes passou por alterações ou não apresenta todos os metadados necessários).

b) Acesso a certos tipos de dados, principalmente em formato matricial como por exemplo imagens de alta resolução que não são disponibilizadas pelas instituições.

c) Acesso aos dados via internet: raramente os órgãos indicam para o usuário, os dados geoespaciais produzidos, e quando indicam, seja através de metadados, ou através de visualização num mapa interativo, geralmente, não permitem que o usuário adquira essa informação pela internet, ou seja, não é possível fazer o download desses dados, principalmente no estado da Bahia.

d) A existência de dados desatualizados, que foi apontada como exemplo a hidrografia do Estado da Bahia. O órgão responsável dispõe apenas desta informação na escala de 1:2.500.000. 
Entretanto nesta escala alguns rios e riachos nem aparecem e outros não tem os nomes cadastrados. Essa situação gera um retrabalho de toda a equipe.

Outra pesquisa foi realizada por Machado e Camboim (2016) com técnicos produtores e usuários de dados espaciais nas instituições relacionadas a gestão da Região Metropolitana de Curitiba (RMC). O estudo identificou que as principais formas de acesso à informação geoespacial são através de arquivos digitais mediante solicitação (CD/DVD) e apenas uma pequena porcentagem de usuários faz através de serviços WMS/WFS. Os principais problemas encontrados na aquisição de dados foram: formatos diferentes; inexistência de dados na escala; qualidade ou atualização necessária; diferentes datas de elaboração; documentação incipiente sobre a metodologia e padrão na produção; políticas de acesso; produção descentralizada com métodos distintos; precisões diversas; inexistência ou não observação de padrões definidos; e desconhecimento de acervos existentes.

Quanto à disponibilização e compartilhamento das informações na RMC foram destacadas: a forma de arquivos digitais mediante solicitação, mapas em papel, portal de acesso interno, página para download, portal de acesso público e serviços WMS/WFS.

Diante desta problemática percebe-se a importância da Infraestruturas de Dados Espaciais para permitir aos usuários esse acesso, compartilhamento e disponibilização de forma eficiente e eficaz. Entretanto pesquisas mostram que a cultura de uso dessas infraestruturas ainda não está disseminada como demonstrado na pesquisa de Lima et al. (2017), que verificou a utilização dos geoserviços da Infraestrutura Nacional de Dados Espaciais (INDE) e da Infraestrutura de Dados Espaciais da Bahia (IDE-BA) por usuários de dados espaciais (profissionais de órgãos públicos da área de geotecnologias e professores de nível superior).

Os questionários aplicados por Lima et al. (2017), com 20 usuários, revelaram que o volume de dados espaciais consumidos pelo público alvo em 
relação aos geoserviços ainda é muito pequeno, estando na faixa de 0 a $33 \%$. Quanto a forma de consumir os dados, percebeu-se que em ambos geoportais a predominância é em relação ao uso da URL para acessar o dado em outra plataforma via WMS/WFS, em detrimento da realização do download. E em relação à experiência no consumo de serviços WMS/WFS, 45\% relatou que considera ter poucos dados disponíveis no geoportal da IDE-BA e 40\% já tiveram dificuldades para obter a URL do serviço no geoportal da INDE.

\section{Metodologia}

A metodologia adotada neste artigo foi baseada em Jesus (2015) e Araújo e Gonzalez (2015), autoras que avaliaram a usabilidade de plataformas computacionais, a exemplo de mapas interativos, visualizadores de mapas e geoportais.

Para avaliar o visualizador de mapas da IDE-Bahia foram elaboradas tarefas com o intuito de avaliar a usabilidade dos principais itens que compõem o Geoportal e o Visualizador de mapas da IDE-BA (conforme Tabela 1), por meio de testes realizados com usuários de informação geoespacial.

Tabela 1 - Tarefas propostas para o teste de usabilidade do geoportal e visualizador de mapas da IDE-BA

\begin{tabular}{c|c}
\hline Tarefa 01 & $\begin{array}{c}\text { Entre no site da IDE Bahia e encontre o visualizador de } \\
\text { mapa do Geoportal. }\end{array}$ \\
\hline Tarefa 02 & $\begin{array}{c}\text { Utilizando a lista de camadas identifique a região do } \\
\text { Recôncavo Baiano. }\end{array}$ \\
\hline Tarefa 03 & $\begin{array}{c}\text { Encontre o município de Cachoeira. Qual a escala de } \\
\text { visualização? }\end{array}$ \\
\hline Tarefa 04 & Calcule a distância entre os municípios de Salvador e \\
Cachoeira.
\end{tabular}

Fonte: Elaborada pelas autoras. 
Desta forma, o teste avaliou ferramentas comumente encontradas em geoportais, dentre elas: a busca de informações no formato de camadas, acesso aos metadados, acesso aos geoserviços WMS e WFS, e visualização dos dados geoespaciais na área do mapa.

Os testes foram aplicados no mês de setembro de 2017 com 6 usuários de informações espaciais (alunos de pós-graduação e professores de ensino superior da Universidade Federal da Bahia). Segundo Nielsen (1993), com uma amostra pequena de usuários, ao menos cinco, já é possível identificar aproximadamente $75 \%$ dos problemas mais críticos de uma interface.

Para o teste foi utilizado um formulário on-line do Google, cujo link foi enviado aos participantes e respondido durante o teste, na presença do avaliador. Este formulário continha informações breves sobre o geoportal da IDE-BA, bem como informava aos participantes que o teste tinha a finalidade de avaliar a interface e não o usuário e que eles deveriam à medida que realizavam as tarefas dizer em que estavam pensando e o que pretendiam fazer (técnica conhecida como Think Aloud). Convém ressaltar que o avaliador acompanhou o usuário durante todo o teste (com o intuito de estimulá-lo) mas, não indicava os passos que deveriam ser seguidos, pois o usuário deveria encontrar as formas de concluir as tarefas, sem auxílio do avaliador.

Ao iniciar o teste de usabilidade, os usuários preencheram informações sobre o perfil de usuários com as seguintes informações: nome, nível de formação, faixa etária, frequência de uso da internet, de uso de mapas e de uso de mapas na web e frequência de uso de dados geoespaciais. Indicavam também se já tinham acessado a IDE, para que finalidade e se a experiência de acesso foi satisfatória.

Em seguida, eram apresentadas as tarefas, que indicavam as ferramentas que deveriam ser utilizadas para concluí-las, e era reservado um campo para o usuário dar as respostas. Ao final de cada tarefa, o mesmo indicava o grau de exigência mental, física e temporal, que a tarefa lhe exigiu 
e também seu desempenho, esforço e insatisfação. Os testes tiveram duração média de uma hora, com cada usuário, individualmente.

O teste foi realizado utilizando os seguintes materiais: um notebook da marca DELL com processador core I5, memória de 8GB, disco rígido de $1 \mathrm{~TB}$, placa de vídeo dedicada de $2 \mathrm{~GB}$; formulário do teste elaborado no google docs; gravador de vídeo do computador e o aplicativo "screenshot captor" que gravava a tela durante as interações do usuário.

Durante o teste para avaliar as interações dos usuários com a interface foram utilizadas as técnicas: gravação de tela e vídeo, observação do pesquisador e thinking aloud. A utilização desta técnica permitiu ao avaliador registrar e questionar, junto ao usuário, possíveis dificuldades enfrentadas e motivos de insatisfações. Também possibilitou verificar se o usuário não iria desistir da tarefa, sem tentar concluí-la, ou não alcançar o objetivo proposto.

Os testes foram analisados a partir do critério da carga de trabalho (adaptado do questionário NASA-TLX) que permite mensurar a satisfação e insatisfação do usuário com a interface em questão e a identificação de questões relacionadas à usabilidade que são percebidas pelo avaliador, durante as interações.

As análises referentes à carga de trabalho foram medidas pela percepção do usuário ao realizar as tarefas do teste, e validadas pela observação do avaliador. Para isso, o usuário julgava o grau de exigência mental, física e temporal, bem como esforço e insatisfação ao realizar ar as tarefas, segundo as opções: muito pouco, pouco, exigente, muito exigente ou extremamente exigente; e para avaliar o desempenho as alternativas eram: perfeito, bom, médio ou ruim de acordo com a Tabela 2.

As seguintes questões relacionadas à usabilidade, foram interpretadas após os testes: ferramentas que foram utilizadas pelo usuário em cada tarefa; dificuldades enfrentadas; motivo que o usuário expressa insatisfação; e tarefa realizada, usando estratégia eficiente e eficaz de trabalho. 
Tabela 2 - Percepção do usuário ao realizar cada tarefa

\begin{tabular}{|c|c|c|}
\hline Exigência & Questionamento & Possíveis Respostas \\
\hline Mental & $\begin{array}{l}\text { Quão mentalmente } \\
\text { exigente foi a tarefa? }\end{array}$ & $\begin{array}{l}\text { ( ) Muito pouco ( ) Pouco ( ) } \\
\text { Exigente ( ) Muito exigente ( ) } \\
\text { Extremamente exigente }\end{array}$ \\
\hline Física & $\begin{array}{l}\text { Quão fisicamente } \\
\text { exigente foi a tarefa? }\end{array}$ & $\begin{array}{l}\text { ( ) Muito pouco ( ) Pouco ( ) } \\
\text { Exigente ( ) Muito exigente ( ) } \\
\text { Extremamente exigente }\end{array}$ \\
\hline Temporal & $\begin{array}{l}\text { Quanta pressão por conta } \\
\text { do tempo dado você } \\
\text { sentiu, para cumprir com } \\
\text { êxito a tarefa? }\end{array}$ & $\begin{array}{l}\text { ( ) Muito pouco ( ) Pouco ( ) } \\
\text { Exigente ( ) Muito exigente ( ) } \\
\text { Extremamente exigente }\end{array}$ \\
\hline Desempenho & $\begin{array}{l}\text { Quão bom foi } \text { seu } \\
\text { desempenho } \\
\text { executar aquilo que lhe } \\
\text { foi solicitado? }\end{array}$ & $\begin{array}{l}\text { ( ) Perfeito ( ) Bom ( ) Médio ( ) } \\
\text { Ruim }\end{array}$ \\
\hline Esforço & $\begin{array}{l}\text { Para chegar ao seu } \\
\text { desempenho, o quanto } \\
\text { você teve que se esforçar? }\end{array}$ & $\begin{array}{l}\text { ( ) Muito pouco ( ) Pouco ( ) } \\
\text { Exigente ( ) Muito exigente ( ) } \\
\text { Extremamente exigente }\end{array}$ \\
\hline Insatisfação & $\begin{array}{l}\text { Quão inseguro, } \\
\text { desencorajado, irritado, } \\
\text { estressado e aborrecido } \\
\text { você esteve ao realizar a } \\
\text { tarefa? }\end{array}$ & $\begin{array}{l}\text { ( ) Muito pouco ( ) Pouco ( ) } \\
\text { Exigente ( ) Muito exigente ( ) } \\
\text { Extremamente exigente }\end{array}$ \\
\hline
\end{tabular}

Fonte: Elaborada pelas autoras.

\section{Resultados e Análises}

Os testes demonstraram que quatro dos seis usuários que participaram do teste já haviam acessado o Geoportal da IDE BA e declararam ter tido uma experiência satisfatória.

Entretanto, os testes revelam alguns problemas de usabilidade que foram identificados pelos usuários e pelo pesquisador/observador. As observações indicaram que:

$\mathrm{Na}$ tarefa 01, todos os usuários conseguiram encontrar rapidamente o visualizador de mapas do Geoportal. Todos os usuários consideraram simples encontrar o visualizador e devidamente posicionado na interface.

$\mathrm{Na}$ tarefa 02 , foi solicitado que os usuários identificassem a região do Recôncavo Baiano utilizando a lista de camadas. Entretanto três usuários acabaram fazendo uma busca no visualizador de mapas, que apenas direciona

Rev. Bras. de Cartografia, vol. 70, Edição Especial "XXVII Congresso Brasileiro de 
a visualização para a região, mas não aparece a divisão da região nem o nome da mesma.

Para visualizar as regiões o usuário deveria encontrar a camada regionalizações e habilitá-la. Entretanto os usuários que tentaram buscar nas camadas demoraram a perceber que a região que foi solicitada estaria dentro da camada Regionalizações e que teriam que habilitá-la para conseguir visualizar o Recôncavo (Figura 2).

Nesta tarefa os motivos de frustração se referiram ao fato de não ser mostrada a área selecionada do Recôncavo Baiano dificultando a identificação dos municípios que o compõem bem como por haver duas opções para habilitar as camadas (torná-la visível e selecionar camada) conforme Figura 3.

Figura 2 - Visualização da área do Recôncavo Baiano.

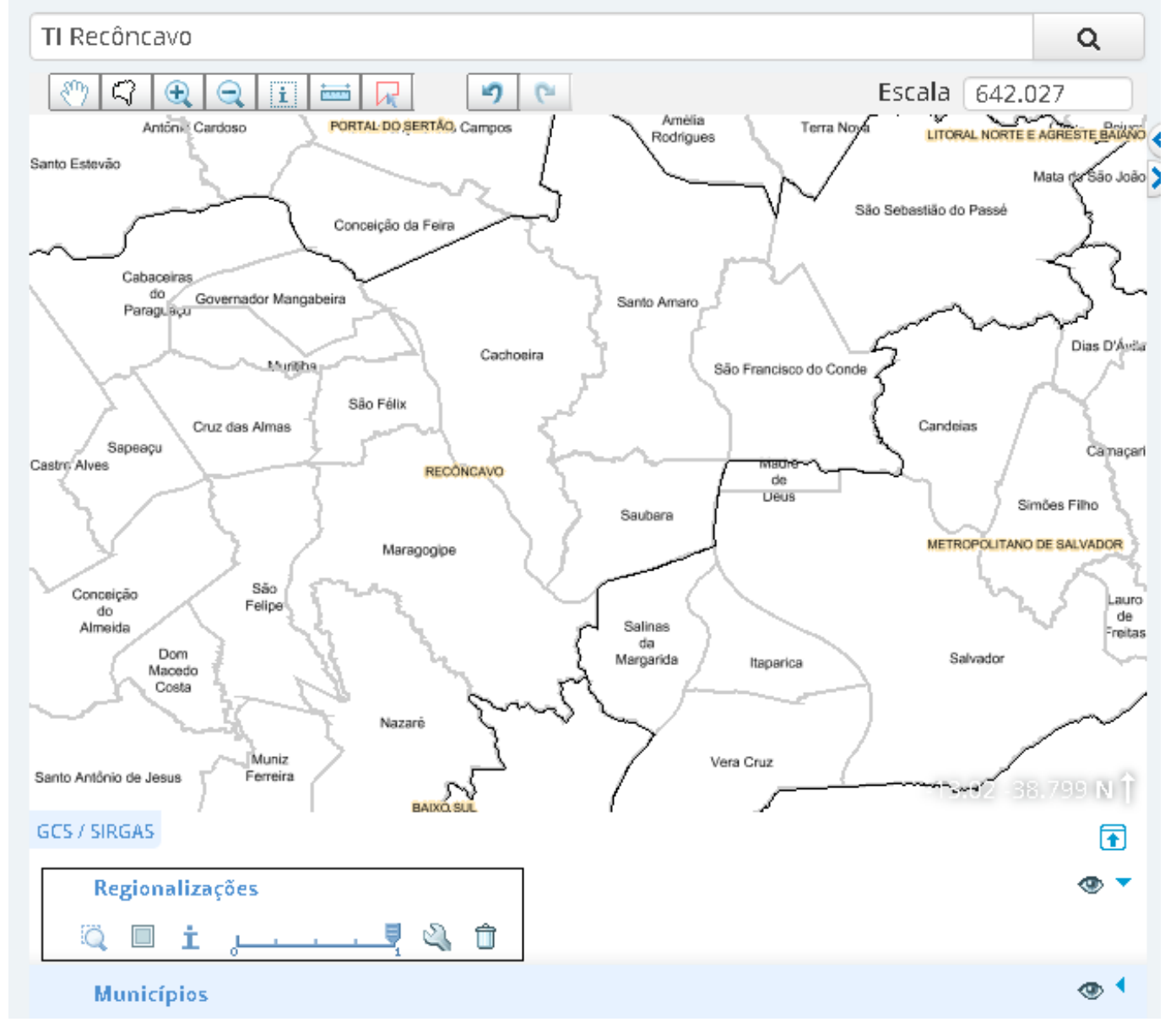

Fonte: IDE-Bahia 
Figura 3 - Opções para habilitar a camada

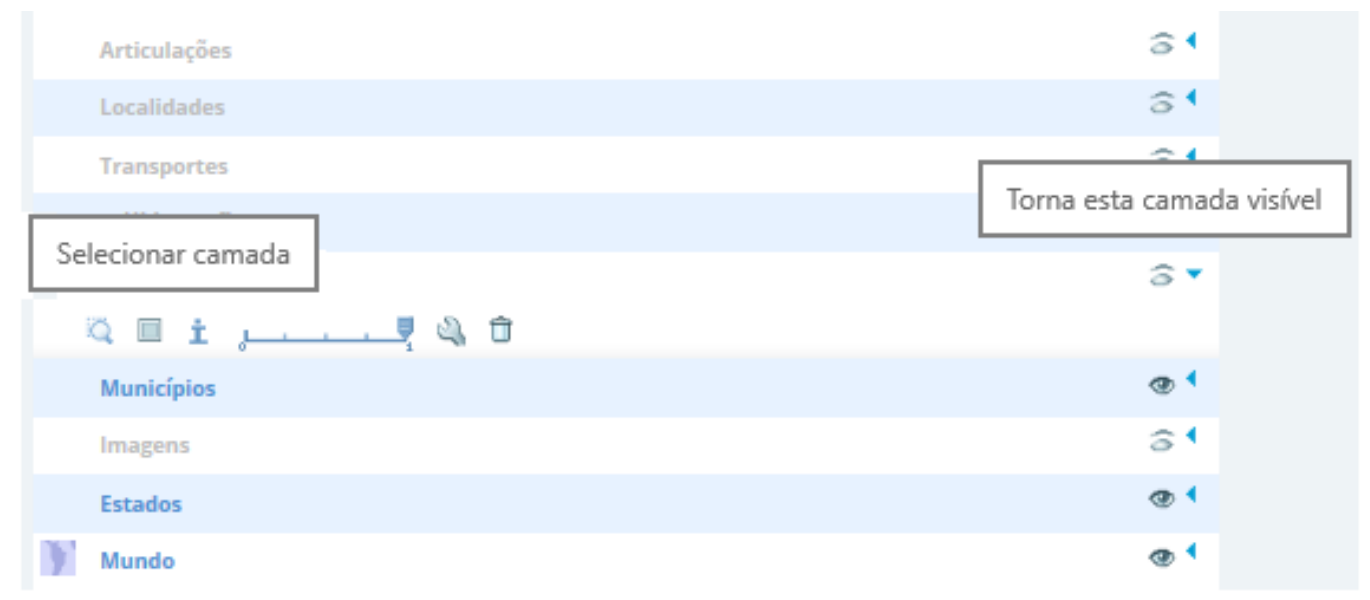

Fonte: IDE-Bahia

$\mathrm{Na}$ tarefa 03, foi solicitado que os usuários encontrassem o município de Cachoeira. E identificassem a escala de visualização. Dos seis usuários, apenas um encontrou visualmente o município sem a ajuda da busca, e dois deles expressaram muita dificuldade pois a ferramenta de busca não levava o usuário diretamente para o município solicitado (conforme verificado na Figura 4).

Entretanto 4 usuários consideraram-se insatisfeitos com a interface pois consideraram que a mesma deveria diferenciar o objeto de busca dos demais e não apenas aproximava para a área. Outro motivo de insatisfação foi a quantidade de informações que eram habilitadas no mapa e impediam a visualização do município tornando o mapa poluído.

Este problema poderia ser solucionado se as camadas não fossem habilitadas automaticamente (com o zoom) e sim a partir da habilitação do usuário. 
Figura 4 - Visualização poluída do mapa devido ao número de camadas

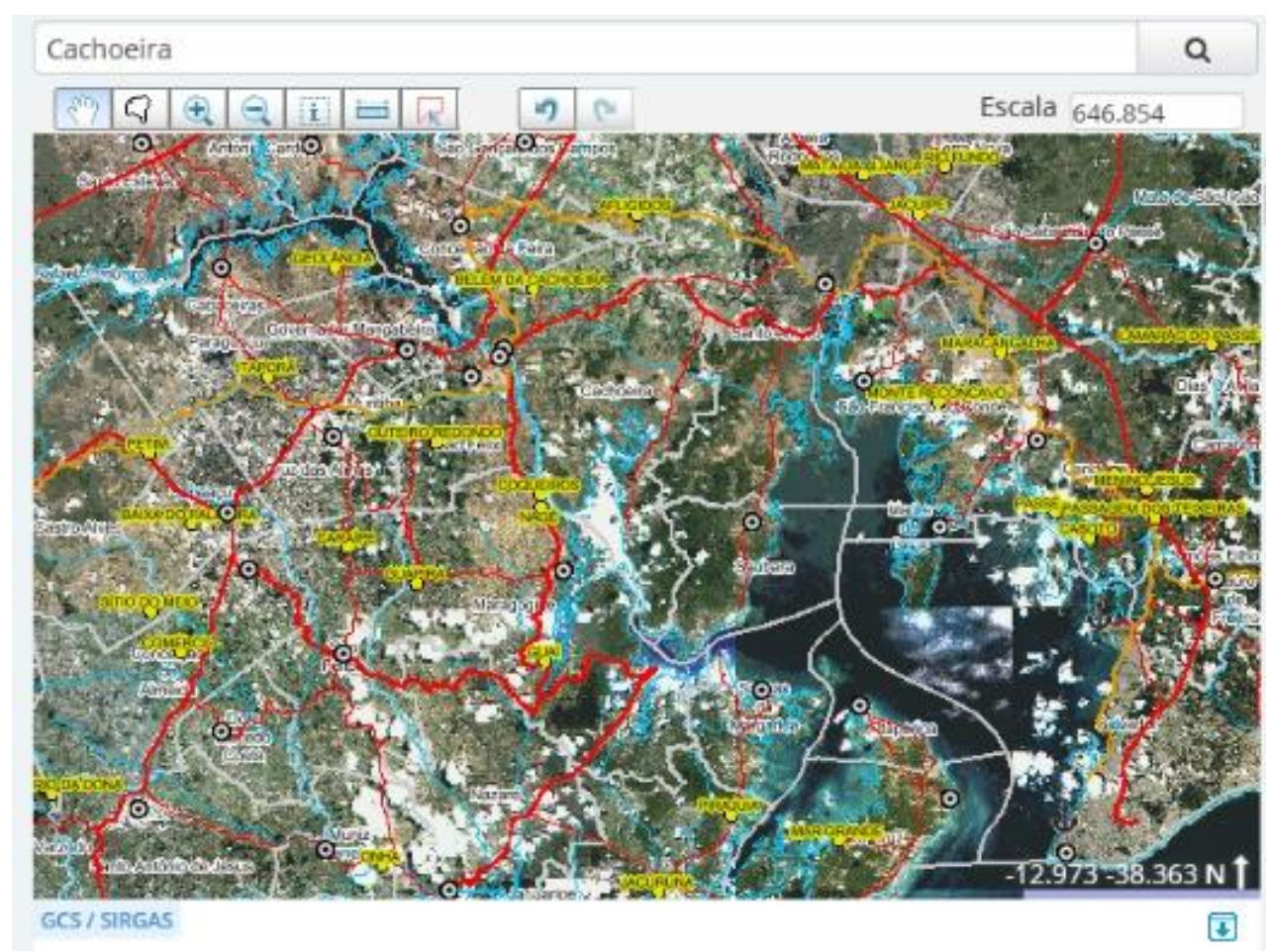

Fonte: IDE-Bahia

$\mathrm{Na}$ tarefa 04 , foi solicitado que realizassem a medição de distância entre os municípios de Salvador e Cachoeira a partir das sedes municipais. Entretanto alguns usuários desconsideraram esta informação e mediram de qualquer ponto dos municípios. Nesta tarefa houve um problema durante o teste de três usuários com a desconfiguração da ferramenta, pois a medida que era iniciada e finalizada a medição a seta era totalmente deslocada e a medição realizada incorretamente (conforme Figura 5), levando-os a desistirem de concluir a tarefa. Dos outros três usuários, 2 tiveram dificuldades para manipular e finalizar a medição, isto é, visualizar os dois municípios simultaneamente, realizar a medição e dar duplo clique para finalizar.

Estes problemas impactaram na satisfação do usuário, resultando em 5 usuários insatisfeitos ao realizar esta tarefa. 
Figura 5 - Desconfiguração da ferramenta de medição de distância

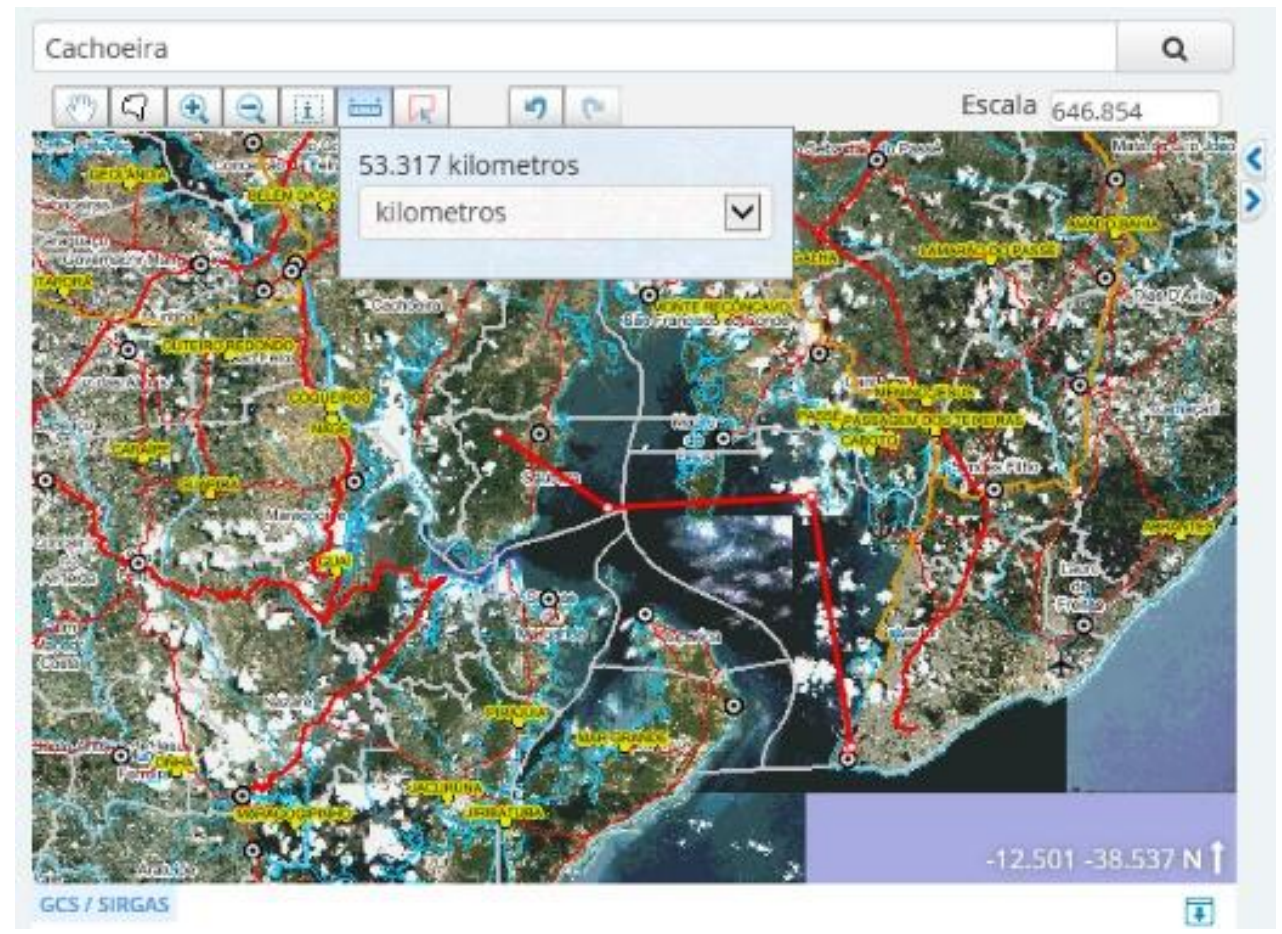

Fonte: IDE-Bahia

$\mathrm{Na}$ tarefa 05, os usuários deveriam realizar uma busca para encontrar o geosserviço WMS da IDE-BA "Eixo de Logradouros do município de Cachoeira" e visualize seus metadados. Entretanto esse geoserviço estava offline e os usuários encontraram outro com o nome "serviço WMS - eixo de logradouro" que compreende vários municípios e estava online, mas não permitia a visualização no mapa (Figura 6).

Nessa tarefa todos os 6 usuários se mostraram insatisfeitos pois questionaram sobre o objetivo de manter geoserviços que não poderiam ser visualizados no mapa.

Rev. Bras. de Cartografia, vol. 70, Edição Especial "XXVII Congresso Brasileiro de 
Figura 6 - Geoserviços que não permitem visualização no mapa

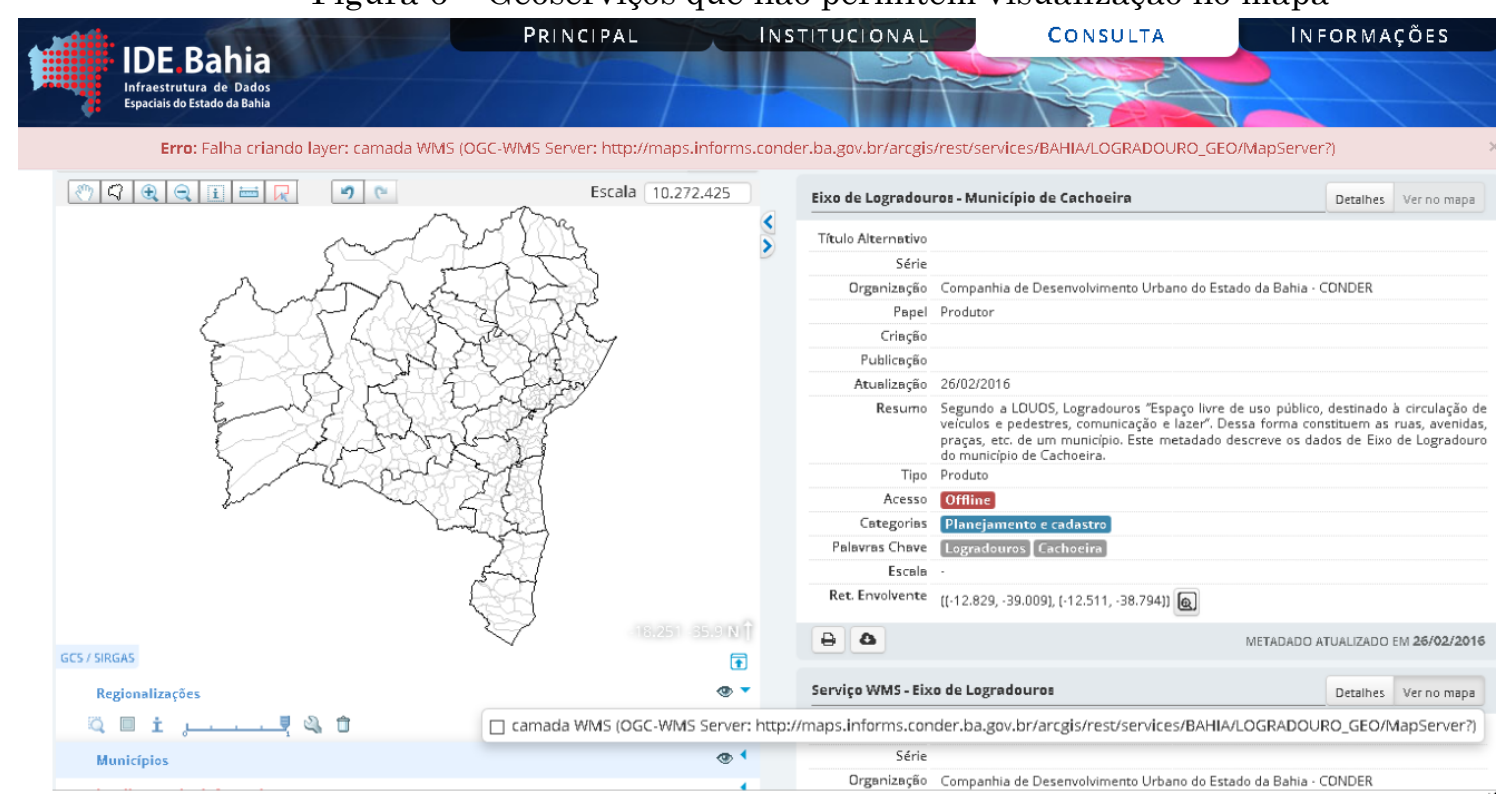

Fonte: IDE-Bahia

$\mathrm{Na}$ tarefa 06, os problemas foram para encontrar o catálogo com acesso a outros órgãos, e em seguida para encontrar algum que estivesse disponível e permitisse a visualização no mapa, pois a maioria não estava disponível como Embrapa, IBGE, MMA, ANA (Figura 7).

Figura 7 - Falha ao conectar o catálogo da Embrapa

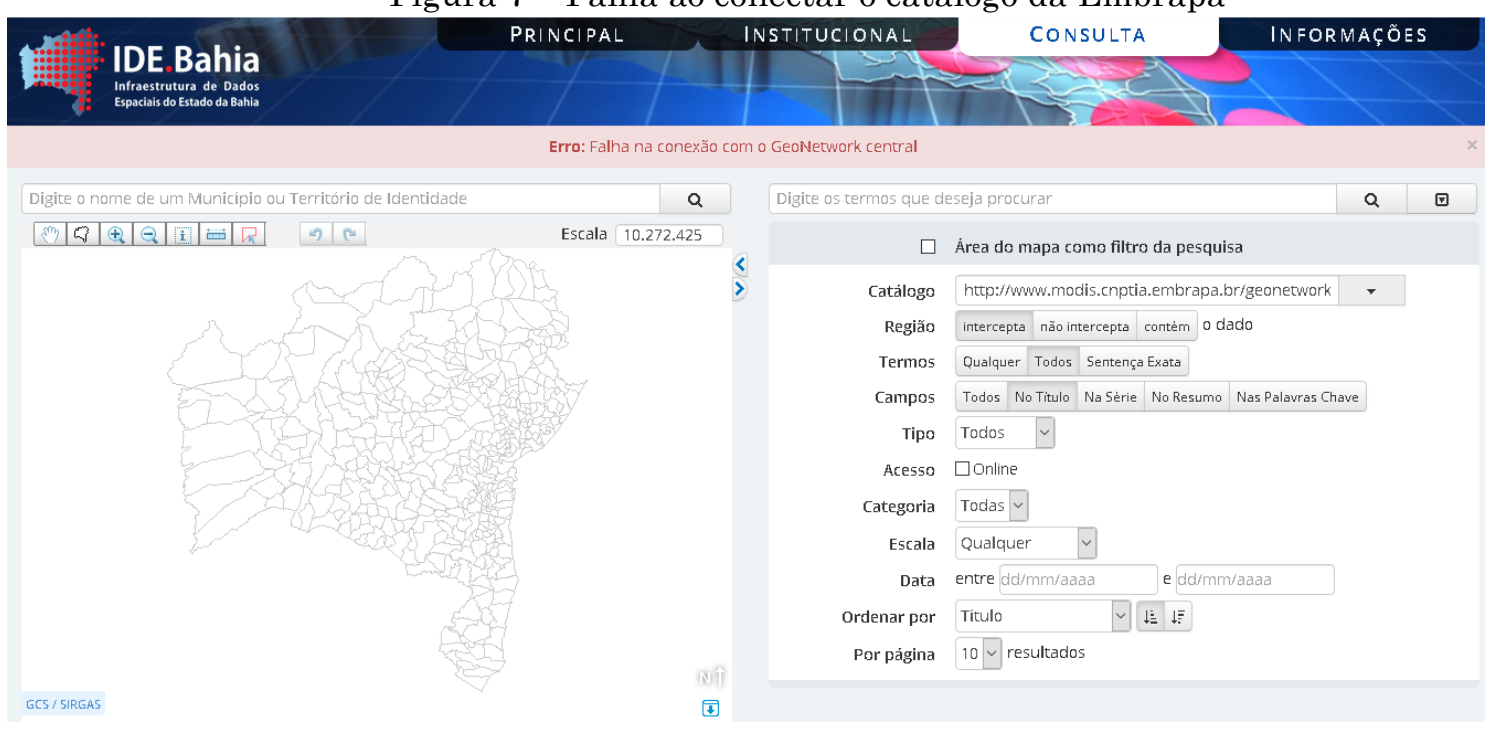

Fonte: IDE-Bahia

Rev. Bras. de Cartografia, vol. 70, Edição Especial "XXVII Congresso Brasileiro de 
$\mathrm{Na}$ tarefa 07 , a legenda do mapa apresenta-se de forma incompleta pois não traz a simbologia da maioria dos itens mostrados no mapa. Enquanto que outros itens como por exemplo os aglomerados subnormais que são mostrados na legenda não aparecem no mapa (Figura 8). Desta forma alguns usuários disseram que não há legenda neste mapa porque não perceberam a simbologia ao lado ou porque não consideraram esta como uma legenda.

Figura 8 - Legenda do mapa

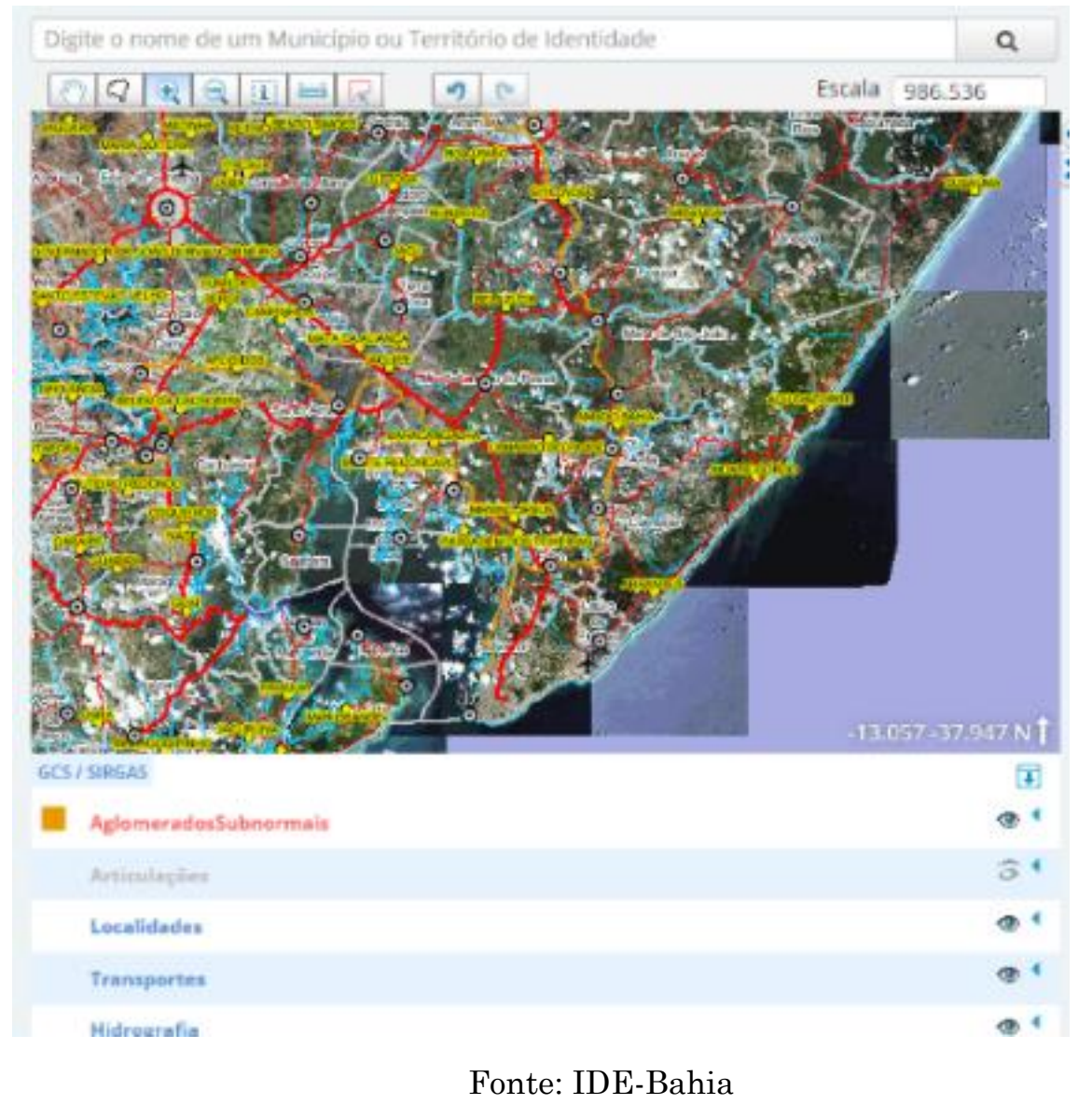

Sendo assim, todos os usuários concluíram as tarefas 01 e 03; $50 \%$ dos usuários não concluíram as tarefas 2, 4 e 5; nenhum usuário conseguiu concluir a tarefa 6 e 66\% dos usuários não concluíram a tarefa 7 . Esses números expressam que devem ser revistas algumas ferramentas do Geoportal pois não estão sendo eficazes e não estão atendendo às necessidades dos usuários.

Rev. Bras. de Cartografia, vol. 70, Edição Especial "XXVII Congresso Brasileiro de 


\section{Considerações Finais}

Diante das análises realizadas percebeu-se que o Geoportal e o visualizador de mapas desta IDE agregam muitos dados geoespaciais que podem ser utilizados pela comunidade de usuários (principalmente técnicos dos setores públicos e privados e a academia).

Os testes de usabilidade demonstraram que muitas ferramentas precisam ser ajustadas como por exemplo a busca na área do mapa, o modo de ativação das camadas, a compatibilidade entre o que é visto no mapa e o que é exibido na legenda e principalmente o acesso aos geoserviços que deveriam possibilitar o acesso dos usuários aos dados geoespaciais de instituições que pertencem a IDE-Bahia e sua conexão com outros órgãos.

Sendo assim, percebe-se o longo caminho que esta Infraestrutura de Dados Espaciais ainda terá que percorrer para conseguir disponibilizar ao usuário os dados padronizados de modo que satisfaça e atenda às necessidades dos mesmos.

\section{Referências}

ARAÚJO, V. O. H.; GONZALEZ, M. E. Usabilidade do Visualizador da Infraestrutura Nacional de Dados Espaciais (VINDE), visão do usuário. Bahia Análise e Dados. Salvador: Superintendência de Estudos Econômicos e Sociais da Bahia, v. 25, n. 4, 2015. pp. 753-772.

BAHIA. Decreto Estadual n 10.185 de 20 de dezembro de 2006. Institui o Portal de Informações Geoespaciais do Estado da Bahia, e dá outras providências. Diário Oficial do Estado da Bahia. Salvador: Poder Executivo, 2006. Site: $<$ http://geoportal.ide.ba.gov.br/geoportal/conteudo/institucional/DECRETO_10 185_Geoportal.pdf>. Acessado em abril de 2018

\section{organizacional}

$\mathrm{n}^{\circ} 13.204$ de 11 de dezembro de 2014

Modifica a estrutura do Poder Executivo Estadual e dá outras providências. Diário Oficial do Estado da Bahia. Salvador: Poder Executivo, 2014. Site: 
$<$ http://www.secom.ba.gov.br/arquivos/File/LEI13204.pdf $>$. Acessado em maio de 2018.

. Decreto Estadual n 16.219 de 24 de julho de 2015. Dispõe sobre a produção, a manutenção, o compartilhamento de dados geoespaciais, seus metadados e sua disseminação, bem como, institui a Infraestrutura de Dados Espaciais da Bahia - IDE-Bahia e dá outras providências. Diário Oficial do Estado da Bahia. Salvador: Poder Executivo, 2015. Site: <http://geoportal.ide.ba.gov.br/geoportal/conteudo/institucional/DECRETO_16 219.pdf $>$. Acessado em abril de 2018.

. Portaria $n^{\circ} 048$ de 1 de agosto de 2017. Homologa o Plano de Ação da Infraestrutura de Dados Espaciais do Estado da Bahia (IDE-Bahia), aprovado pela Comissão Estadual de Cartografia e Geoinformação (CECAR) em 26 de outubro de 2016 que poderá ser acessado através do Geoportal IDE Bahia. Diário Oficial do Estado da Bahia, Salvador, 02 de ago. de 2017.

CAMBOIM, S. P. Arquitetura para Integração de Dados Interligados Abertos à INDE-BR. Curitiba: Universidade Federal do Paraná. 140 f. Tese (Doutorado em Ciências Geodésicas) - Programa de Pós-Graduação em Ciências Geodésicas, Setor de Ciências da Terra, Universidade Federal do Paraná, 2013.

DIAS, CLÁUDIA. Usabilidade na WEB: criando portais mais acessíveis. Editora Alta Books, Rio de Janeiro, 2003.

GSDI - Global Spatial Data Infrastructure Association. The SDI Cookbook, Version 2.0. 2004. Site: <http://www.gsdi.org/gsdicookbookindex>. Acessado em maio de 2018.

ISO - INTERNATIONAL ORGANIZATION FOR STANDARDIZATION. ISO 19115: geographic information- metadata. Genève, Switzerland, 2003a. 98p.

JESUS, E. G. V. Análise de usabilidade de mapas interativos nas atividades de ensino superior. Salvador: Universidade Federal da Bahia. $187 \mathrm{f}$. Dissertação (Mestrado em Engenharia Ambiental Urbana), Universidade Federal da Bahia. 2015.

LIMA, L.; JESUS, E. G. V.; BRITO, P. L. Utilização de geoserviços nas infraestruturas de dados espaciais. In: Simpósio Regional de Geoprocessamento e Sensoriamento Remoto (Geonordeste), 8., 2017, Salvador. Anais... Aracaju: Rede Sergipe de Geotecnologias, 2017. 
LISBOA FILHO, J.; VEGI, L. F. M.; SOUZA, W. D.; LAMAS, J. P. C.; COSTA, G. L. S.; OLIVEIRA, W. M.; CARRASCO, R. S.; FERREIRA, T. G.; BAIA, J. W. Uma infraestrutura de dados espaciais para o projeto GeoMINAS com metadados definidos no perfil MGB da INDE. Revista Brasileira de Cartografia. Rio de Janeiro: Sociedade Brasileira de Cartografia, Geodésia, Fotogrametria e Sensoriamento Remoto, n. 65, v. 1, 2013, pp. 123-138.

MACHADO, A. A.; CAMBOIM, S. P. Diagnóstico da perspectiva do usuário na criação de infraestruturas de dados espaciais subnacionais: estudo de caso para a Região Metropolitana de Curitiba. Revista Brasileira de Cartografia. Rio de Janeiro: Sociedade Brasileira de Cartografia, Geodésia, Fotogrametria e Sensoriamento Remoto, n. 68, v. 8, 2016, pp. 1633-1651.

NASA. Task Load Index (TLX): computerized version (Version 1). Califórnia: Human Research Performance Group, 1986.

NIELSEN, J. Usability Engineering. EUA: AP PROFESSIONAL, 1993.

NOGUERAS-ISO, J.; ZARAZAGA-SORIA, F. J.; MURO-MEDRANO, P. R. Geographic information metadata for spatial data infrastructures. New York: Springer, 2005. 263p.

PAIXÃO, S.; NICHOLS, S.; and COLEMAN, D.,Towards A Spatial Data Infrastructure: Brazilian Initiatives. Revista Brasileira de Cartografia. 60(2), August 2008. Site: <http://www.rbc.ufrj.br/_2008/60_2_04.htm>. Acessado em novembro de 2017.

SOUZA, F. A; SILVA, F. S; SILVA, H.R.F. Cadastro de metadados geoespaciais: experiencia no contexto da Infraestrutura de Dados Espaciais do Estado da Bahia. Bahia Análise e Dados. Salvador: Superintendência de Estudos Econômicos e Sociais da Bahia, v. 25, n. 4, 2015, pp. 753-772.

SOFTWARE USABILITY MEASUREMENT INVENTORY (SUMI). Site: < http://sumi.ucc.ie/>. Acessado em junho de 2018.

WEBSITE ANALYSIS AND MEASURE MENT INVENTORY (WAMMI). Site: $<$ http://www.wammi.com/>. Acessado em junho de 2018.

WINCKLER, M. A.; PIMENTA, M. S. Avaliação de Usabilidade de Sites Web, em Escola de Informática da SBC Sul (ed) Sociedade Brasileira de Computação (SBC), Porto Alegre, pp. 85-137, 2002. 